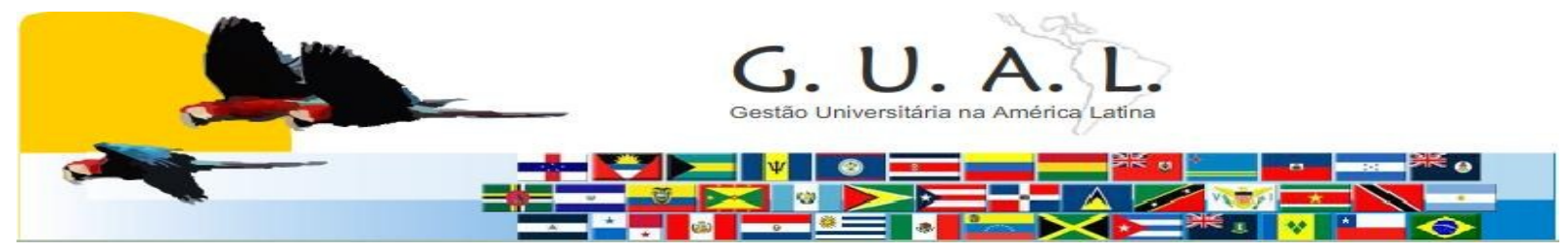

ISSN 1983-4535

\title{
EXPANSÃO DO INSTITUTO FEDERAL DE SANTA CATARINA: A IMPLANTAÇÃO DO CAMPUS GAROPABA E SUA RELAÇÃO COM O MERCADO
}

\author{
Tiago Savi Mondo, Mestre \\ Instituto Federal de Santa Catarina - IFSC \\ tiago.mondo@ifsc.edu.br
}

\begin{abstract}
RESUMO
A expansão no Instituto Federal de Santa Catarina é realidade, onde de 3 campi passou a contar com 19 campi. O Campus Garopaba é um dos que está em implantação. As dificuldades são grandes o a parceria com o mercado da região é pressuposto básico para o sucesso desta implantação. Dessa forma, o objetivo deste artigo é verificar os mecanismos de relacionamento e comunicação com o mercado que ocorrem no processo de implantação do Campus Garopaba do Instituto Federal de Santa Catarina a partir de pesquisa participante. O estudo é caracterizado como exploratório-descritivo, do tipo relato de caso. Foi realizada pesquisa participante e de observação para descrição das atividades no Campus. Os resultados demonstram que o Campus utiliza mecanismos de mídia impressa, rádio, reuniões com autoridades e associações e a internet e mídias digitais. Considera-se importante as iniciativas de relacionamento e de comunicação em processo de ida e volta, realizando-se dessa maneira uma construção coletiva, juntamente com a comunidade.
\end{abstract}

Palavras-chave: IFSC. Campus Garopaba. Relacionamento com o mercado. Comunicação. 


\section{INTRODUÇÃO}

O Instituto Federal de Santa Catarina, organização com 102 anos de história, vem realizando nos últimos cinco anos um processo de expansão. Em 2005 eram 3 campi (Florianópolis, São José e Jaraguá do Sul) e atualmente possui 19 campi, alguns ainda em implantação, como é o caso do Campus Garopaba.

O processo de expansão possibilitou à organização, novos desafios e perspectivas e fez com que o pensamento gestor se ampliasse e o IFSC fosse considerado uma organização universitária.

A Universidade de acordo com Borges e Araújo (2001) é uma estrutura administrativa, um sistema politizado, um complexo científico que denota justamente a complexidade e a multidiversidade.

Colossi (1999) complementa mencionando que a Universidade é uma organização única, movendo-se em um ambiente altamente dinâmico, sendo também influenciada constantemente pela crescente democratização da sociedade, bem como pelo aumento substancial da problemática social e tecnológica, que acompanha e caracteriza a vida humana na sociedade. Toda esta complexidade intrínseca da Universidade, a impossibilita de funcionar nos mesmos paradigmas de outros tipos organizacionais, impedindo-a também de responder, adequadamente, às questões de controle e avaliação, essenciais para reordenação da missão e objetivos institucionais.

Como salienta Melo (2008) a Universidade necessita estabelecer uma interface mais dinâmica com toda a sociedade, assumindo que sua responsabilidade vai além da formação de profissionais para atendimento das necessidades do mercado. Ela deve propor ações que propiciem o desenvolvimento de novos produtos e serviços que melhorem a qualidade de vida da sociedade.

Dessa maneira, este artigo terá como foco a relação com o mercado da região de Garopaba a partir do processo de implantação do Campus do IFSC no município.

Trindade (1999) menciona que as Universidades são fortemente orientadas por premissas de mercado, sendo que a oferta está focada na expectativa da sociedade, seja pelo seu próprio perfil comercial ou vocação regional.

Assim, as Universidades devem se preocupar com os lados externos, com o mercado em que atua, justamente para a definição do perfil do profissional egresso, dos cursos e 
conteúdos que deverão ser oferecidos e as informações base para isso advém dos contatos com o empresariado e governo (DESIDERIO, 2004).

Taborga e Di Marco (2001) complementam a questão ao afirmarem que existem possibilidades, quando se percebe o mercado, de estender e potencializar as oferta através da incorporação de novas figuras e estruturas. Um exemplo disso seria a formação de conselhos dentro da Universidade com a participação de membros do mercado de trabalho.

A partir disso, estar alinhada ao segmento empresarial se torna pressuposto básico para a manutenção da qualidade da oferta de cursos em uma Universidade. Um mecanismo para manter tal alinhamento é uma comunicação eficaz, que proporcione explorar especificidades, desde as lógicas procedentes do mercado, até o desenvolvimento do sistema de educação, mostrando a multidimensionalidade presente nesta questão

Baseado no exposto, o objetivo deste artigo é verificar os mecanismos de relacionamento e comunicação com o mercado que ocorrem no processo de implantação do Campus Garopaba do Instituto Federal de Santa Catarina a partir de pesquisa participante.

\section{REFERENCIAL TEÓRICO}

\subsection{Gestão universitária}

A origem da primeira universidade surgiu na missão de transformar a sociedade através do conhecimento do potencial humano, sendo suas funções ensinar, pesquisar e exercer atividades na comunidade (OSPINA, 1990).

Uma das mais importantes características diferenciais da universidade com relação a outras organizações é a de que ela é formada de pessoas para pessoas. Nela se transmitem e se absorvem símbolos através dos quais tendem a influenciar o meio social. O que a caracteriza, é a produção de símbolos, idéias, conhecimentos, valores (VLASMAN e CASTRO, 1985).

Atualmente a universidade vem passando por transformações, e está sendo chamada a desenvolver um papel diferente do tradicional. As mudanças que têm ocorrido e as Universidades são condicionadas à apresentarem melhores resultados, custos administrativos e operacionais menores, gerando um aluno bem mais qualificado para enfrentar o mercado de trabalho. Preservar e renovar são dilemas dessa força conservadora ou de transformação da sociedade (MONÇÃO, 2000). 
Para Finger (1993) a área da administração universitária preocupa-se com a problemática da gestão organizacional em instituições de ensino superior. Geralmente, a administração universitária é classificada em duas modalidades: os serviços administrativos e de infra-estrutura (atividade meio) e a prática acadêmica que compreende laboratórios, prática de estágios, professor em sala de aula, etc (atividade fim).

Considerando a responsabilidade da universidade neste terceiro milênio com a manutenção ou incorporação de novos mecanismos de aprimoramento ao ensino e à pesquisa, certamente esses precisam ser melhor estudados e analisados com critérios seletivos. Neste caso, buscar conhecer com intensidade a realidade do mundo empresarial, para que os benefícios adquiridos com o conhecimento resultante possam intervir em favor da melhoria do ensino e da formação do profissional, do desenvolvimento tecnológico das empresas é o mínimo de preocupação que a universidade deverá ter (NOVO e MELO, 2004).

Desiderio(2004) menciona que é necessário um novo modo de pensar, agir e se impor no ambiente universitário: preocupação com o mercado, com o negócio, com o cliente e com a gerência de todos os serviços oferecidos.

Gestores universitários e a sociedade em geral estão promovendo intensos debates questionando a verdadeira missão da universidade, a quem ela deve atender quais são as suas funções primordiais, como deverá portar-se perante as mudanças de mercado, enfim, discussões que dizem respeito ao futuro de seus egressos, bem como ao seu próprio futuro como instituição (NOVO e MELO, 2004). Assim um novo processo é criado, a relação entre Universidade e Mercado.

\subsection{Relação com o mercado}

As universidades vêm passando por vários processos de transformação, conjuntamente com a chamada era do conhecimento. Nesta fase evolutiva e no cenário em que estão inseridas, provavelmente necessitam de novas técnicas e métodos de gestão. Acima de tudo, elas devem focar no profissional que está formando para o mundo do trabalho, suas habilidades, perfil, competência, dentre outras características que propiciem um diferencial frente ao mercado de trabalho (TEIXEIRA, 2005).

Tachizawa e Andrade (2002, p. 58) afirmam que "o ambiente externo ou mercado é um fator contingencial que estabelece parâmetros, limites, propostas e desafios a serem 
interpretados e tornados significativos, de acordo com a escala de valores vigentes na instituição".

A maioria das Universidades reconhece as novas exigências e a real necessidade de adaptação e muitas delas já tem consciência do grande desafio a ser enfrentado, sendo um dos pontos o equilíbrio externo que são as novas expectativas que o mercado tem em relação ao trabalho que as Universidades terão para ajudar os novos profissionais a estar mais bem preparados para o novo mercado de trabalho (SANTURIO, 2000).

A experiência vivenciada por outros países tem evidenciado que a cooperação Universidade-Segmento empresarial é um fenômeno crescente e constitui forte instrumento de promoção da inovação tecnológica e do desenvolvimento em geral (BRASIL/MCT, 2002)

O processo de cooperação Universidade-segmento empresarial pode trazer inúmeros benefícios, tantos para os agentes envolvidos diretamente quanto para a sociedade. Dentre os benefícios da cooperação Universidade-segmento empresarial, pode-se afirmar que a empresa ganha, pois obterá o retorno de seu investimento e a universidade, porque alcança seu objetivo de tornar disponível à sociedade os resultados de sua pesquisa (STAL et. al., 2006).

Assim, os benefícios da cooperação ultrapassam a universidade e o segmento empresarial, e se estendem ao país por meio de um setor industrial mais competitivo, pesquisas de melhor qualidade, setor acadêmico com maior estabilidade na execução de pesquisas, mais rápidas inovações tecnológicas e fortalecimento da economia nacional (MENDES e SBRAGIA, 2002).

Melo (2008) explicita como uma das dificuldades o fato de não se ter uma regra estabelecida, um padrão que pudesse ser seguido para que seja realizado o processo de cooperação. Ele aponta como uma das causas disso, o fato de na universidade ter-se uma cultura de descentralização por área e uma maximização do poder constituído, onde as unidades acadêmicas e administrativas possuem interesses particulares e uma relativa autonomia no processo decisório. Isso faz com que o processo de cooperação seja realizado de forma isolada, onde cada centro de ensino, departamento, laboratório desenvolva seus projetos.

Dessa maneira, apresenta-se, conforme Melo (2008), os requisitos fundamentais para o processo de cooperação Universidade-Segmento empresarial alinhado com os princípios e finalidades da universidade: sistema de comunicação; formação do corpo técnico- 
administrativo, docente e dirigentes; reestruturação do ensino; legislação e acompanhamento; criação de fundos de incentivo à pesquisa; propriedade intelectual a patentes; avaliação institucional; incentivo ao desenvolvimento de pesquisas; laboratórios; e ações governamentais.

A cooperação entre Universidade e Mercado somente acontece e se legitima quando existe um processo de comunicação bem delineado e exposto.

\subsection{Comunicação universidade mercado}

Santurio (2000) menciona que o mercado de trabalho, as empresas e os profissionais estão mudando em função das novas exigências e dessa forma, a adaptação às mudanças de mercado deve ser/continuar como base informacional para a formação desses profissionais.

Para Finger (1993) em um ambiente globalizado, marcado pela exigência de competitividade, agilidade, flexibilidade e qualidade de informação, as organizações têm buscado apoio no uso de sistemas de informações executivas, para tomada de decisões estratégicas. As instituições universitárias brasileiras, neste contexto, também deveriam usar da mesma ferramenta para apoiar suas decisões.

As universidades estão inseridas em contextos especialmente ricos, que ensejam múltiplas possibilidades de contribuição à sociedade, desde suas necessidades básicas e vitais, até o desenvolvimento de tecnologias de ponta, gerando assim, a melhoria da qualidade de vida da população. Portanto, não basta somente à universidade gerar novos conhecimentos, deve fazer chegá-los a sociedade embutidos em produtos e serviços que proporcionem a população uma melhoria em suas condições sociais (MELO, 2002).

De acordo com Araújo, Pereira e Oliveira ( 2010) a informação, o conhecimento, a tecnologia e as pessoas devem caminhar juntas para que um sistema de comunicação e conhecimento aconteça. De acordo com Choo (2003) deles dependem os processos de criação de significado e construção do conhecimento para tomada de decisão.

Para Santos (2004), a gestão, a qualidade e a velocidade da informação são essenciais à competitividade econômica. As tecnologias de informação e de comunicação têm a característica de não só contribuírem para o aumento da produtividade, mas também de serem incubadoras de novos serviços onde a educação assume lugar de destaque.

Rev. GUAL., Florianópolis, v. 4, n. 3, p.213-227, set/dez. 2011 
Necessária tanto para ajudar a identificar problemas quanto para solucioná-los, a informação torna-se um recurso e como tal deve ser tratada com características especificadas e mensuráveis, como método de coleta, uso, ciclo de vida padrão, com diferentes atributos em cada estágio. Pode também ser transformada em produtos que possibilitam à organização atingir seus objetivos. (TEIXEIRA, 2005).

Santurio (2000) apresenta alguns benefícios da aproximação de contatos entre mercado e Universidade. São eles: Acesso à tecnologia de ponta com baixo investimento; serviços de pesquisa e assessoria com mão de obra qualificada e baixo custo; apóio na implantação de novos negócios, pesquisas, planejamento e instalação; aumentar as chances de sucesso nos processos de recrutamento; redução dos investimentos no treinamento inicial do jovem profissional; atrair e reter jovens talentos para a empresa.

\section{PROCEDIMENTOS METODOLÓGICOS}

Tão ou mais relevante quanto definir os objetivos e problema de pesquisa, é a definição do delineamento da pesquisa que será utilizado, principalmente pelo fato de que ambos estão estreitamente relacionados. (FERNANDES e GOMES, 2003).

Segundo seus objetivos, este artigo envolve pesquisa exploratória-descritiva. De acordo com Gil (2002) a pesquisa exploratória tem como objetivo proporcionar maior familiaridade com o problema, buscando torná-lo mais explícito ou a construir hipóteses. Busca-se através delas o aprimoramento das ideias ou a descoberta de intuições. Tal fato é corroborado por Malhotra (2006) que menciona que a pesquisa exploratória tem como objetivo explorar ou realizar uma busca em um problema. Segundo Zikmund (2006), como o nome indica, a pesquisa descritiva tem a função de descrever as características de uma população ou de um fenômeno.

A pesquisa é qualitativa. Esta, segundo Godoi e Balsini (2006) abrange diversas formas de pesquisa e auxilia o pesquisador a compreender e explicar o fenômeno social imerso no ambiente. Creswell (2007) complementa mencionando que o pesquisador tenta estabelecer o significado de um fenômeno a partir do ponto de vista dos participantes da pesquisa. O comportamento dos participantes deve ser observado. 
A pesquisa qualitativa é fundamentalmente interpretativa. Significa dizer que o pesquisador interpreta os dados a partir da descrição de um fato ou cenário por uma pessoa. (CRESWELL, 2007)

A coleta de dados foi iniciada com pesquisas documentais e bibliográficas, com o intuito de obter todos os dados secundários possíveis que possam auxiliar na elaboração da pesquisa e em sua discussão.

A pesquisa participante e de observação foram os métodos de coleta de dados escolhidos para a pesquisa. A pesquisa participante insere-se na pesquisa prática, classificação apresentada por Demo (2000, p.21), para fins de sistematização. Segundo esse autor, a pesquisa prática "é ligada à práxis, ou seja, á prática histórica em termos de usar conhecimento científico para fins explícitos de intervenção; nesse sentido, não esconde sua ideologia, sem com isso necessariamente perder de vista o rigor metodológico". Há na pesquisa participante um componente político que possibilita discutir a importância do processo de investigação tendo por perspectiva a intervenção na realidade social.

Dessa forma este artigo caracteriza-se por um relato de caso, apresentando as principais ações utilizada pelo Campus Garopaba na sua relação e comunicação com o mercado.

\section{RESULTADOS}

\subsection{O processo de expansão do IFSC}

No dia 29 de dezembro de 2008, pela Lei 11.892/2008 foi instituída a Rede Federal de Educação Profissional, Científica e Tecnológica e criados 38 Institutos Federais de Educação, Ciência e Tecnologia.

A Lei 11.892/2008 é um desdobramento do Decreto 6095/2007 e da Chamada Pública 02 de 12/12/2007. A partir desses dois documentos, foram realizados debates abertos com toda a comunidade para apresentar os motivos que levaram a defesa para transformação do CEFET-SC em Instituto Federal. Naquela oportunidade houve a proposição de uma tese favorável e de uma tese contrária.

Após a realização de 18 debates, a comunidade aprovou a transformação por $76 \%$ dos votos. O CEFET-SC foi o único do país a realizar consulta a sua comunidade para tomada de decisão sobre essa transformação. Após envio do Projeto de Transformação para a SETEC e 
sua aprovação, prontamente iniciou-se o processo de construção do novo PDI - Plano de Desenvolvimento Institucional. Isso se deu em julho de 2008.

Legalmente, o IF-SC é uma instituição de educação superior, básica e profissional, pluricurricular e multicampi que tem por finalidade formar e qualificar profissionais no âmbito da educação tecnológica, nos diferentes níveis e modalidades de ensino, para os diversos setores da economia, bem como realizar pesquisa aplicada e promover o desenvolvimento tecnológico de novos processos, produtos e serviços, em estreita articulação com os setores produtivos e a sociedade, especialmente de abrangência local e regional, oferecendo mecanismos para a educação continuada.

O IF-SC tem como órgão executivo a Reitoria, composta por 1 (um) Reitor e 5 (cinco) Pró-Reitores. A relação entre a Reitoria e os campi é definida com clareza no Regimento Interno. Tem ainda como órgãos superiores o Colégio de Dirigentes e o Conselho Superior. O Colégio de Dirigentes, de caráter consultivo, é composto pelo Reitor, pelos Pró-Reitores e pelo Diretor-Geral de cada um dos campi que integram o Instituto Federal de Santa Catarina. O Conselho Superior, de caráter consultivo e deliberativo, é composto por representantes dos docentes, dos estudantes, dos servidores técnico-administrativos, dos egressos da instituição, da sociedade civil, do Ministério da Educação e do Colégio de Dirigentes do Instituto Federal de Santa Catarina, assegurando-se a representação paritária dos segmentos que compõem a comunidade acadêmica.

\subsection{A criação e implantação do Campus Garopaba}

O Campus Garopaba foi criado a partir da audiência pública realizada em 30/07/2010 na cidade onde foram definidos também a construção civil e hospitalidade e lazer como eixos precursores dos cursos oferecidos.

A partir disso, o Campus começou a ser estruturado em uma sala comercial alugada e as aulas oferecidas em parceria com uma escola estadual. Os servidores foram chamados por concurso e atualmente o Campus conta com 2 técnicos administrativos e 7 professores.

Com a definição dos eixos de atuação os professores do Campus puderam de alguma maneira trabalhar com bases sólidas de conteúdo e possibilitar a oferta de cursos necessários para a capacitação profissional da sociedade.

Rev. GUAL., Florianópolis, v. 4, n. 3, p.213-227, set/dez. 2011 
De início foram e ainda são oferecidos cursos de Formação Inicial e Continuada (FIC) como forma de estar presente na comunidade enquanto um curso técnico não é oferecido.

Foram oferecidos em quase dois anos de atuação 12 cursos FIC e certificadas mais de 200 pessoas em diferentes áreas de atuação. O Curso Técnico, previsto para início no segundo semestre de 2012 será na área da hospedagem, pela demanda crescente de qualificação profissional para os meios de hospedagem da região.

Dessa forma, o Campus Garopaba tem se estruturado internamente de forma a possibilitar à população da região oportunidades de qualificação e capacitação.

\subsection{Ações de comunicação e relacionamento com o mercado}

Visando a integração com a região e principalmente com o mercado o Campus Garopaba investe em algumas alternativas de forma a intensificar o relacionamento e promover uma comunicação eficaz com o empresariado, buscando envolvê-lo e torná-lo parceiro da Instituição.

Este artigo apresenta estas ações, com uma análise de sua eficácia e os pontos que necessitam de melhorias.

1. Informativo quinzenal:uma das maneiras de relacionamento com o mercado é o informativo quinzenal organizado pelos professores com notícias sobre as ações do Campus na comunidade e do IFSC de forma geral no âmbito estadual. Esse informativo tem tido um retorno interessante, segundo avaliação dos professores, tem mostrado à população de Garopaba, que apesar da sede física não estar construída ainda, existem ações sendo realizadas e justificando a existência do Campus na região.

2. Parceria com a Associação Comercial e Industrial de Garopaba-ACIG: esta parceria tem trazido bons frutos para o Campus. Primeiramente, pelo auxílio da Associação na divulgação dos cursos para os empresários vinculados e depois por todas as possibilidades e recursos demandados pela Associação. Existe atualmente um curso FIC de Espanhol Básico sendo oferecido nas dependências da associação.

3. Entrevistas nas rádios da cidade: Como forma de melhorar a comunicação e atingir o maior número de pessoas possíveis na região, os professores frequentemente visitam a rádio da cidade para entrevistas, divulgação de cursos e outras questões que surgem.

4. Palestras para o empresariado: Como forma de integração com o empresariado, já foram realizadas 4 palestras pelos professores do Campus que trataram de temáticas desafiadoras para o empresariado local, como o planejamento e o diagnóstico situacional. As palestras tem sido uma boa alternativa de verificar junto aos 
empresários as principais necessidades de capacitação para seus funcionários e para eles próprios.

5. Newsletter para o empresariado hoteleiro: Como o curso técnico que será oferecido é na área da hospedagem, o Campus buscou uma aproximação com o empresariado do ramo. Semanalmente, a partir de Novembro de 2011 será enviada uma newsletter com notícias da hotelaria no mundo, Brasil e Santa Catarina.

6. Participação nos eventos da Cidade: Os professores do Campus identificaram que a integração com a comunidade iria aumentar se estivessem presentes como participantes e algumas vezes auxiliando na organização. Dessa forma, o Campus participou em mais de 30 eventos no ano de 2011, em diferentes temáticas como Educação Ambiental e meio ambiente, encontros sobre história da região, encontros dos Conselhos Municipais de Turismo e Meio Ambiente, reuniões de Associações como a ACIG e a AMA (Associação do Meio Ambiente), Festas populares em toda a região (Garopaba, Imbituba, Paulo Lopes e Imarui) e outros tipos de eventos.

7. Presença nas mídias sociais. O Campus também mantém um perfil ativo no Facebook, incluindo todas as atividades que são realizadas e todas as atividades que os professores e técnicos participam. Tem-se tido sucesso nesta ação. A comunidade e o empresariado tem acompanhado e interagido com o perfil na rede social o que é avaliado como positivo.

\section{CONCLUSÃO}

Este artigo buscou verificar os mecanismos de comunicação e relacionamento do Campus Garopaba do IF-SC com o empresariado local a partir de uma pesquisa de observação participante.

Visualiza-se a partir do exposto nos resultados que o Campus realiza tentativas de comunicação em diversas frentes como forma de atingir o empresariado local. Considera-se relevante esse caminho de idas e vindas, a percepção dos anseios do empresariado e o fechamento do ciclo com a oferta de cursos que de alguma maneira poderão suprir as necessidades de capacitação do mercado empresarial da cidade.

A comunicação e o relacionamento estão intimamente ligadas com a percepção de qualidade e valor que toda a comunidade tem de uma instituição. Estar atento a tal fato, fez com que o Campus buscasse agir em mídias impressas (informativo), mídias auditivas (rádio) e mídias digitais (redes sociais e emails). 
Além disso, salienta-se a participação do Campus em eventos na região, fazendo com que a comunidade geral identifique a Instituição e valorize sua marca como sendo de ensino gratuito, público e qualidade.

Dessa forma, percebe-se que outras ações deverão ser realizadas, como a criação de um Conselho Universitário no Campus e Grupos temáticos para discussão da oferta de cursos, bem como a maior participação em eventos e o aumento da amplitude geográfica de atuação.

Entretanto, é relevante mencionar que as ações de comunicação e relacionamente tem tido sucesso, e os empresários tem avaliado como positiva a postura do Campus em estar a disposição para ouvi-los e comunicá-los do que acontece.

O caminho é longo, as ações deverão se intensificar. O processo de implantação ainda está no inicio, mas é visível que a comunidade e o empresariado de Garopaba aceitou, legitimou e é parceiro do IFSC na cidade na busca de uma gestão mais eficaz e consequentemente uma educação de maior qualidade para todos da região.

\section{REFERENCIAS}

ARAÚJO, P. PEREIRA, S. OLIVEIRA, M. Compartilhamento de informação e Conhecimento. Revista ACB: Biblioteconomia em Santa Catarina, Florianópolis, v.15, n.1, p. 244-259 jan./jun., 2010.

BORGES, Djalma Freire; ARAÚJO, Maria Arlete Duarte. Uma experiência de planejamento estratégico em universidade: o caso do centro de ciências sociais aplicadas da UFRN. Revista de Administração Pública. FGV. Rio de Janeiro 35 (4) 63-76 jul/ago 2001.

BRASIL. Ministério da Ciência e Tecnologia. Livro Branco: ciência, tecnologia e inovação. Brasília: Ministério da Ciência e Tecnologia, 2002

CHOO, W. C. Organização do conhecimento: como as organizações usam a informação para criar significado, construir conhecimento e tomar decisões. São Paulo: Senac, 2003.

COLOSSI, N. Controle da gestão na universidade: algumas considerações. Encuento Regional Noa Sobre Avaluación De La Educación Superior. Anais..Universidad Nacional de Tucumán. San Miguel de Tucumán, Argentina, 1999.

COOPER, Donald R.; SCHINDLER, Pamela S. Métodos de pesquisa em administração. 7 ed. Porto Alegre: Bookman, 2003

CRESWELL, J. Projeto de pesquisa: métodos qualitativo, quantitativo e misto. 2. ed. Porto Alegre: Bookman, 2007. 
DESIDERIO, M. Desafios da Gestão Universitária. Faculdades DOM BOSCO. 2004.

FERNANDES, L. A.; GOMES, J. M. M. Relatórios de pesquisa nas ciências sociais: características e modalidades de investigação. Contexto, Porto Alegre, v. 1, p. 71-92, 2003.

FINGER A P. A gestão universitária no Brasil - in gestão das universidades - novas abordagens. Curitiba: Champagnat, 1997.

FINGER, A. P. Construindo programas acadêmicos com qualidade total. Florianópolis: IEPES, 1993.

GIL AC. Como elaborar projetos de pesquisa. São Paulo (SP): Atlas; 2002.

GODOI, C. K.; MATTOS, P. L. C. L. de. Entrevista qualitativa: instrumento de pesquisa e evento dialógico. In: GODOI, C. K.; MELO, R. B. de; BARBOSA, A. Pesquisa Qualitativa em Estudos Organizacionais - Paradigmas, Estratégias e Métodos. São Paulo: Saraiva, 2006.

GODOI, C; BALSINI, C. A pesquisa qualitativa nos estudos organizacionais brasileiros: uma análise bibliométrica. In: SILVA, A. et al (orgs) Pesquisa qualitativa em estudos organizacionais. São Paulo: Saraiva, 2006.

KANAANE, R. Comportamento humano nas organizações: o homem rumo ao século XXI’São Paulo: Atlas, 1995.

MALHOTRA, Naresh K. Pesquisa de marketing. Porto Alegre: Bookman, 2006.

MCDANIEL, C., \& GATES, R. Pesquisa de Marketing. São Paulo: Pioneira Thomson Learning, 2003.

MELO, Pedro Antônio de. A cooperação universidade/empresa nas universidades públicas brasileiras. 2002. Tese (Doutorado em Engenharia de Produção) Programa de PósGraduação em Engenharia de Produção, Universidade Federal de Santa Catarina, Florianópolis, 2002.

MELO, Pedro Antônio de.. A autonomia universitária e seus reflexos na gestão e nos resultados de universidades brasileiras. In. LANER, Aline dos Santos. CRUZ JÚNIOR, João Benjamim da (Org). Indivíduo, organizações e sociedade. Ijuí: Unijuí, 2008.

MENDES, Andréia Paula Segatto; SBRAGIA, Roberto. O processo de cooperação universidadeempresa nas universidades brasileiras. Revista de Administração. São Paulo v.37, n.4, p.58-71, outubro/dezembro 2002.

MONÇÃO NETO, J. R. Atividades administrativas, serviços de apoio, produção acadêmica: um diálogo necessário na reformulação estrutural da universidade, 2000. 
NOVO, L.F.; MELO, P.A., Universidade empreendedora: fortalendo os caminhos para a responsabilidade social. In: de MELO, Pedro Antonio.; COLOSSI, Nelson. (Org). Cenários da gestão universitária na contemporaneidade. Florianópolis: Insular, 2004. cap.1, p.1735 .

NUNES, G. LANZER, E. SERRA, F. Abordagem do Marketing de Relacionamento no Ensino Superior: Um Estudo Exploratório. IN: VI Colóquio Internacional Sobre Gestão Universitária Na América Do Sul, Blumenau, 2006.

OSPINA, G. L. Definição de uma agenda para o ensino superior nos anos 90. In: Crub. Universidade, Estado e Sociedade na década de 90. Brasília, 1990.

ROTHENBÜHLER, Renata. Universidade Empreendedora. Florianópolis, 2000. 135f. Dissertação (Mestrado) Programa de Pós-graduação em Engenharia de Produção. UFSC, 2000.

SANTOS, Boaventura de Sousa. A universidade no século XXI: para uma reforma democrática e emancipatória da universidade. São Paulo: Cortez, 2004. 120p.

SANTURIO, P. Núcleo de Integração Universidade-Mercado. In: I Colóquio sobre gestão universitária na América do Sul, 2000.

STAL, Eva et al. Inovação: como vencer este desafio empresarial. São Paulo: Clio Editora, 2006.

TACHIZAWA, T., ANDRADE, R. O. B. Gestão de instituições de ensino. 3 ed. Rio de Janeiro: Fundação Getúlio Vargas, 2002.

TECCHIO et AL. Cooperação universidade-segmento empresarial: dificuldades e mecanismos facilitadores do processo. In: X Colóquio sobre gestão universitária na América do Sul. Mar Del Plata, 2010.

TEIXEIRA, Darlene. Informação utilizada nos processos decisórios de Gestores universitários: estudo de caso na puc-campinas, sp. 2005. 130f. Dissertação (Mestrado em Ciência da Informação) - Pontifícia Universidade Católica de Campinas, Campinas, 2005.

TRINDADE, H. Universidade em ruínas: na república dos professores. Petrópolis/RJ: Vozes, 1999.

VLASMAN, P. M.; CASTRO, L. F. M. Universidade: a luta pelo poder. Revista Brasileirade Administração da Educação. Porto Alegre, v. 3, p. 88-101, jan./jun. 1985.

ZIKMUND, William G. Princípios da Pesquisa de Marketing. São Paulo: Pioneira Thomsom Learning, 2006. 


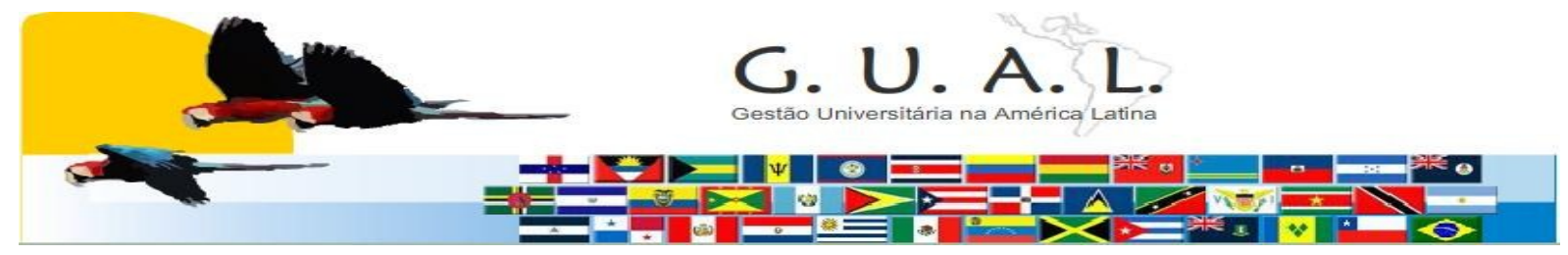

ISSN 1983-4535

\title{
EXPANSION OF FEDERAL INSTITUTE OF SANTA CATARINA: IMPLEMENTATION OF CAMPUS GAROPABA AND ITS RELATION TO THE MARKET
}

\author{
Tiago Savi Mondo, Master \\ Instituto Federal de Santa Catarina - IFSC \\ tiago.mondo@ifsc.edu.br
}

\begin{abstract}
The expansion at the Federal Institute of Santa Catarina is reality, where the three campuses now has 19 campuses. And Campus Garopaba is one that is under construction. The difficulties are great partnership with him to market the region's basic assumption for the success of this deployment. Thus, the purpose of this paper is to examine the mechanisms of relationship and communication with the market occurring in the process of implementation of the Campus Garopaba of Federal Institute of Santa Catarina from research participant. The study is characterized as exploratory and descriptive type case report. Survey was conducted participant observation for a description of activities on campus. The results show that the mechanisms Campus uses print, radio, meetings with authorities and associations and the Internet and digital media. It is considered important initiatives of relationship and communication in the process of return, thereby carrying out a collective construction, together with the community.
\end{abstract}

Keywords: IFSC. Campus Garopaba. Relationship marketing. Communication. 